Benchmarks

\title{
Homogenous M13 bacteriophage quantification assay using switchable lanthanide fluorescence probes
}

Ari Lehmusvuori, Julius Manninen, Tuomas Huovinen, Tero Soukka, Urpo Lamminmäki

Department of Biochemistry and Food Chemistry, Division of Biotechnology, University of Turku, Finland

BioTechniques 53:301-303 (November 2012) doi 10.2144/0000113954

Keywords: switchable fluorescence, lanthanide chelate, bacteriophage quantification, time-resolved measurement, filamentous phage

We have developed a rapid and reliable bacteriophage quantification method based on measurement of phage single-stranded DNA (ssDNA) using switchable lanthanide chelate complementation probes. One oligonucleotide probe contains a non-fluorescent lanthanide ion carrier chelate and another probe is labeled with a light absorbing antenna ligand. Hybridization of the non-fluorescent complementation probes in adjacent positions on the released bacteriophage ssDNA leads to high local concentrations of the lanthanide ion carrier chelate and the antenna ligand, inducing formation of a fluorescent lanthanide chelate complex. This method enables monitoring of bacteriophage titers in a $20 \mathrm{~min}$ assay with a dynamic range of $10^{9}-10^{12} \mathrm{cfu} / \mathrm{mL}$ in a microtiter well format. While designed for titering filamentous bacteriophage used in phage display, our method also could be implemented in virological research as a tool to analyze ssDNA virus reproduction.

Switchable lanthanide chelate complementation probe technology is a versatile tool for homogenous DNA detection assays due to low background fluorescence levels and high specific signal generation. Complementation probe technology allows detection of low picomolar quantities of target DNA in a homogenous DNA hybridization assay (1) and enables remarkably high signalto-background ratio (S/B) (maximum of 300) in a closed-tube PCR assay (2). The technology involves the use of two non-fluorescent oligonucleotide probes, one carrying a lanthanide ion and the other, an antenna probe. When hybridized to a DNA target at precisely adjacent positions, the lanthanide ion and antenna probe form a highly fluorescent lanthanide chelate complex.
Phage display (3) is a widely used technique for finding binders by screening large heterologous peptide or protein populations. In phage display, a library of genes is fused to a phage coat protein and expressed on the surface of virus particles. Since the genotype and phenotype are linked by the virion, the displayed protein or peptide with the desired binding characteristics can be easily propagated and isolated. The virus used in phage display is the M13 filamentous bacteriophage of Escherichia coli; in fact, the M13 bacteriophage based phage display is the most successful selection technology used in in vitro antibody development (4).

Phages selected are then propagated after each selection (panning) round using a bacterial host. Starting from a universal antibody library, three panning rounds are commonly performed to enrich for the desired target binding antibodies. After each propagation cycle, phage concentration must be determined for better control of the panning process. Phage titers are most often determined by using the plating method, that is the gold standard for the technique. Here, E. coli cells are first infected with a dilution series of the produced phage and then either mixed in soft agar and plated, or plated on top of selective agar containing antibiotics. Following overnight incubation at $37^{\circ} \mathrm{C}$, either plaques (plaque forming units or pfu) or colonies ( colony forming units or cfu) are counted (5). Since phage consists of DNA and proteins that absorb light at the UV region, UV photometry at $265 \mathrm{~nm}$ wavelength is also commonly used to measure phage titers (6). Although titering by absorbance is a rapid technique compared with plating, very pure phage stocks are needed since protein impurities interfere with the measurement. For minimal impurities, phage should be purified using $\mathrm{CsCl}$ density-gradient ultracentrifugation, which requires centrifugation times up to $36 \mathrm{~h}(7)$.

We have developed a rapid mix-andmeasure method for determining phage titer directly in the culture medium, as well as from purified phage preparations. The method is based on measurement of M13 phagemid single stranded DNA (ssDNA) with complementation probe technology and detects ssDNA rather than infectious particles. Therefore, it is important that the standard phage used be the same as, or as similar as possible, to the phage being measured so the amounts of non-infective virus or undetected phage do not influence the results.

Probes were prepared by labeling one 24-mer oligonucleotide (5'-TAT ACGCAAGGCGACAAGGTGCT G(AmC6)-3', Thermo Fisher Scientific, Waltham, MA, USA) at the $3^{\prime}$ end with the non-fluorescent heptadentate $7 \mathrm{~d}$-DOTA-Eu ${ }^{\mathrm{III}}$ carrier chelate (2,2',2''-(10-(3-isothiocyanatobenzyl)1,4,7,10-tetraazacyclo-dodecane-1,4,7 triyl)tri(acetato)europium $\left.{ }^{\mathrm{III}}\right)(8)\left(\mathrm{Eu}^{\mathrm{III}}\right.$ probe) and another 24-mer oligonucleotide (5'-(AmC6)TGCCGCTGGCGATTCAGGTTCA-3', Thermo Fisher Scientific) at the $5^{\prime}$ end with the tridentate light absorbing Antenna ligand (4-((4-isothiocyanatophenyl) ethynyl)pyridine-2,6-dicarboxylic acid) (1) (Antenna probe). The 7d-DOTA$\mathrm{Eu}^{\mathrm{III}}$ and Antenna ligand containing 
A

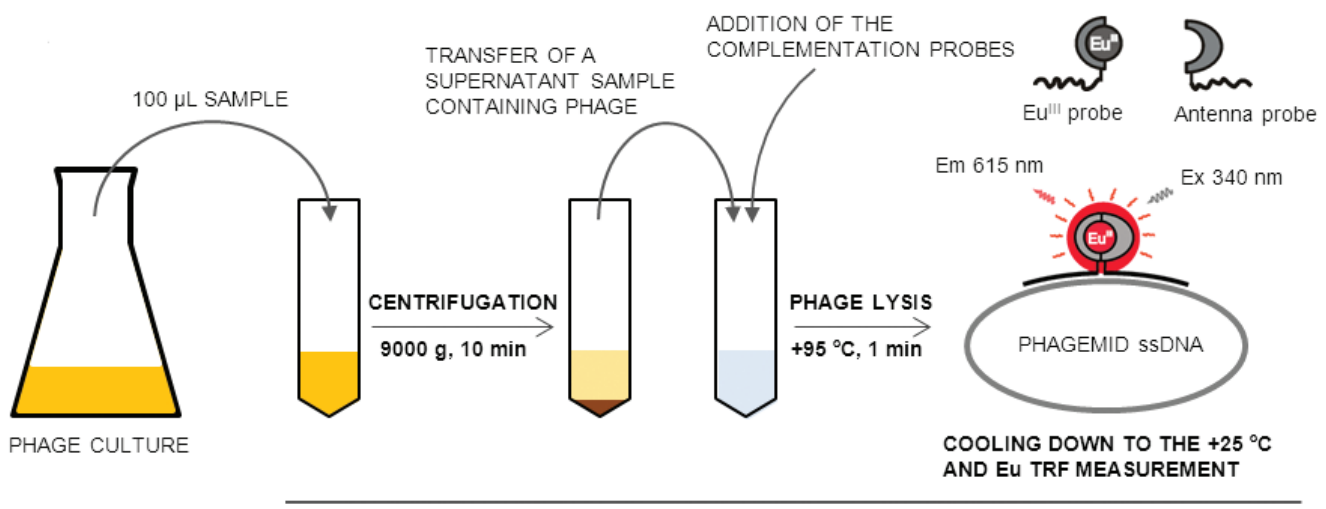

B

Total assay time $20 \mathrm{~min}$<smiles>O=C(O)CNCCN(CCN(CCN(CC(=O)O)CC(=O)O)CC(=O)O)Cc1cccc([N+](=O)[O-])c1</smiles>

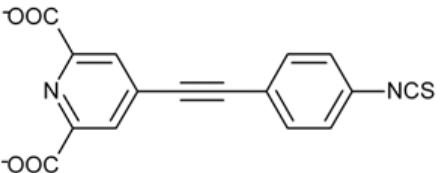

Figure 1. Principle of the complementation probe-based bacteriophage quantification assay. (A) Bacteriophage are isolated from bacteria by centrifugation and complementation probes are added to the solution. Then phagemid SsDNA is released by lysing the phage at high temperatures and Europium timeresolved fluorescence is measured at $+25^{\circ} \mathrm{C}$. (B) Molecular structures of the 7d-DOTA-Eu'l' carrier chelate (1) and the light absorbing Antenna ligand (2). The isothiocyanate group (NCS) is used for coupling the reporter molecules to the oligonucleotides.

A

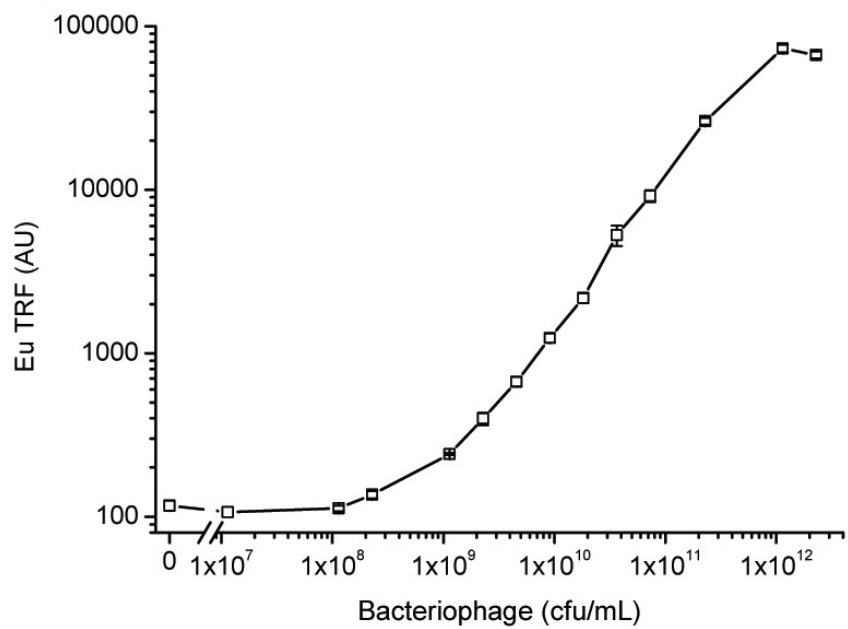

B

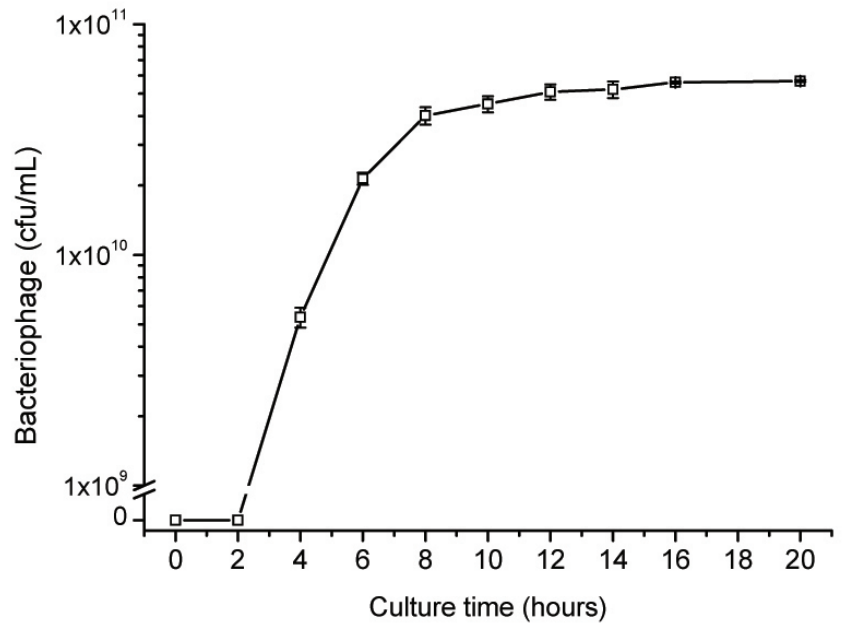

Figure 2. Performance of the complementation probe-based bacteriophage quantification assay. Complementation probes hybridize to the released bacteriophage ssDNA and form a fluorescent lanthanide chelate complex. (A) Phage assay standard curve prepared using purified scFv-phage. (B) Concentration of the scFv-phage in the culture media was monitored during $20 \mathrm{~h}$ culture.

isothiocyanate-groups were coupled to the free amino groups of the carbon linkers attached to the oligonucleotides, forming a covalent thiourea bond as described in Karhunen et al. (1). The probes were designed to hybridize to adjacent positions on the anti-sense strand of the chloramphenicol acetyltransferase gene, a selectable marker on the phagemid pEB91 (9), packed into the M13 phage with VCSM13 helper phage (Stratagene, La Jolla, CA, USA). Hybridization of the probes leads to a high local concentration of the reporter molecules (7d-DOTA-Eu ${ }^{\mathrm{III}}$ and Antenna ligand), inducing formation of a fluorescent mixed chelate complex by self-assembly of the reporter molecules. Principles of the assay and reporter molecule structures are shown in Figure 1.

A stock of M13 bacteriophage displaying an anti-lysozyme singlechain fragment variable (scFv) antibody (amL3/4-42-pEB91-clone, Kd: $0.8 \mathrm{nM}$ ) isolated from the single-framework library, published by Brockmann et al. (9), was prepared according to the 
protocol described by Korpimäki et al. (10) using VCSM13 and E. coli XL-1 blue from Stratagene. Infective titer $(\mathrm{cfu} / \mathrm{mL})$ of the prepared $\mathrm{scF}$-phage stock was determined by the plating method based on antibiotic selection and colony counting. A standard curve was created by measuring a dilution series of the scFv-phage that covered $0-2.7 \times 10^{12} \mathrm{cfu} / \mathrm{mL}$ using the complementation probe assay. The reaction mixture consisted of $10 \mathrm{nM} \mathrm{Eu}{ }^{\mathrm{III}}$ probe, $5 \mathrm{nM}$ Antenna probe and scFv-phage in assay buffer $(25 \mathrm{mM}$ TRIS-HCl, $900 \mathrm{mM} \mathrm{NaCl}, 0.1 \%$ Tween 40, 0.05\% $\mathrm{NaN}_{3}, 30 \mu \mathrm{M}$ DTPA at $\left.\mathrm{pH} 7.5\right)$. The phage were lysed at $95^{\circ} \mathrm{C}$ for $1 \mathrm{~min}$ and the reaction mixture was cooled $1-3^{\circ} \mathrm{C} /$ min followed by $5 \mathrm{~min}$ incubation at $+25^{\circ} \mathrm{C}$ before measurement. Europium time-resolved fluorescence (Eu TRF) was measured using a $340 \mathrm{~nm}$ excitation filter, $615 \mathrm{~nm}$ emission filter, $0.4 \mathrm{~ms}$ delay, and $0.4 \mathrm{~ms}$ measurement time with a 1420 Victor Multilabel Counter (Perkin Elmer Life and Analytical Life Science, Turku, Finland).

The detection limit of the standard, defined as the lowest measurement point giving higher signal than the background $+3 \times$ SD of the background signal, was $1.14 \times 10^{9} \mathrm{cfu} / \mathrm{mL}$. The average coefficient of variation of all standard curve measurement points (three individual assays with three replicas) was $6.4 \%$, indicating reliable measurement performance. When the scFv-phage titer on the standard curve exceeded the concentration of the probes, the signal started to decrease because the probes were hybridized to different scFv-phage ssDNA molecules. (Figure 2A)

The titer of the emerging scFvphage was also monitored directly in the culture medium. A sample was withdrawn from the phage culture and prepared as described in Figure $1 \mathrm{~A}$ every two hours during $20 \mathrm{~h}$ phage culture. Prepared samples were diluted 1:7 in assay buffer containing $10 \mathrm{nM}$ and $5 \mathrm{nM} \mathrm{Eu}{ }^{\mathrm{III}}$, and Antenna probes, respectively. Eu TRF of the reactions was measured (three parallel reactions at each measurement point) and converted to $\mathrm{cfu} / \mathrm{mL}$ according to the phage standard curve. At $4 \mathrm{~h}$, the phage titer had already exceeded the detection limit; the maximum titer was reached by $8-10 \mathrm{~h}$ (Figure $2 \mathrm{~B}$ ). Only $20 \mathrm{~min}$ was needed for measuring the titer at each measurement point.

Phage quantification with complementation probes is a robust method for titer determination, even when measurements are performed directly in culture supernatant. It drastically decreases the total assay and hands-on time compared to the plating method and gives more accurate counts of the phagemid-containing virions than absorbance measurements since contaminating proteins and helper phage do not interfere with the assay. In addition, variation in absorbance measurements can occur since the length of the phage filament is dependent on the size of the packed phagemid; this difficulty is avoided when using the complementation assay. Since the complementation probe based phage assay measures ssDNA directly, the assay should be applicable to quantification of any ssDNA phage species.

\section{Acknowledgments}

This work was supported by the National Doctoral Program of Advanced Diagnostic Technologies and Applications (DIA-NET), the Instrumentarium Science Foundation, and by the Academy of Finland [grant no. 132007].

\section{Competing interests}

Authors Dr. Lamminmäki and Prof. Soukka are inventors on patent application WO2010/109065 relating to assay methods based on the switchable lanthanide luminescence.

\section{References}

1. Karhunen, U., L. Jaakkola, Q. Wang, U. Lamminmaki, and T. Soukka. 2010. Luminescence switching by hybridization-directed mixed lanthanide complex formation. Anal. Chem. 82:751-754.

2. Lehmusvuori, A., U. Karhunen, A.H. Tapio, U. Lamminmaki, and T. Soukka. 2012. High-performance closed-tube PCR based on switchable luminescence probes. Anal. Chim. Acta 731:88-92.

3. Smith, G.P. 1985. Filamentous fusion phage: novel expression vectors that display cloned antigens on the virion surface. Science 228:1315-1317.

4. Schirrmann, T., T. Meyer, M. Schutte, A. Frenzel, and M. Hust. 2011. Phage display for the generation of antibodies for proteome research, diagnostics and therapy. Molecules 16:412-426.

5. Sambrook, J., E.F. Fritsch, and T. Maniatis. 1989. Molecular cloning a laboratory manual (2nd ed.). Cold Spring Harbor, NY: Cold Spring Harbor Laboratory.

6. Denhardt, D.T., D. Dressler, and D.S. Ray. 1978. The single-stranded DNA phages. Cold Spring Harbor Laboratory Press, Cold Spring Harbor, N.Y.

7. Wickner, W. 1975. Asymmetric orientation of a phage coat protein in cytoplasmic membrane of Escherichia coli. Proc. Natl. Acad. Sci. USA 72:4749-4753.

8. Karhunen, U., J. Rosenberg, U. Lamminmaki, and T. Soukka. 2011. Homogeneous detection of avidin based on switchable lanthanide luminescence. Anal. Chem. 83:9011-9016.

9. Brockmann, E.C., S. Akter, T. Savukoski, T. Huovinen, A. Lehmusvuori, J. Leivo, O. Saavalainen, A. Azhayev, et al. 2011. Synthetic single-framework antibody library integrated with rapid affinity maturation by VL shuffling. Protein Eng. Des. Sel. 24:691-700.

10. Korpimäki, T., J. Rosenberg, P. Virtanen, T. Karskela, U. Lamminmäki, M. Tuomola, M. Vehniäinen, and P. Saviranta. 2002. Improving broad specificity hapten recognition with protein engineering. J. Agric. Food Chem. 50:4194-4201.

Received 21 September 2012; accepted 18 October 2012.

Address correspondence to Ari Lehmusvuori, Tykistökatu 6A, 20520 Turku, Finland. Email: artule@utu.fi

To purchase reprints of this article, contact: biotechniques@fosterprinting.com
$2 \mathrm{H} 8$ - The Clone You Want. anti-DYKDDDDK ( $\mathrm{N}$-term) affinity tag monoclonal antibody. Extreme sensitivity. Lowest background. Flow, ICC, IF, IP, WB. Detects what others can't on cells and membranes. Great for GPCRs. Another unique biotool brought to you by...

COSMO BIO CO., LTD. Inspiration for Life Science
North America www.cosmobiousa.com $760-431-4600$ info@cosmobiousa.com
All other locations www.cosmobio.com +81-3-5632-9617 export@cosmobio.co.jp 